\title{
Line Shape of Emission Spectra of the Luminescent Polymer Poly( $p$-Phenylene Vinylene)
}

\author{
A. Marletta, F. E. G. Guimarães, and R. M. Faria \\ Instituto de Física de São Carlos, Universidade de São Paulo, \\ Caixa Postal 369, CEP 13560-970, São Carlos - SP, Brazil
}

Received on 5 December, 2001

\begin{abstract}
The influence of electron-phonon coupling in the emission spectra of self-assembly (SA) films of poly ( $p$-phenylene vinylene) (PPV) is discussed. PPV is an important polymeric system because it exhibits strong luminescence effect. A semi-empirical model was used to analyze the photoluminescence spectra of PPV. This model assumes that defects along the molecule give rise to a distribution of conjugated segments of different lengths. In addition, it was considered that electronic transition was essentially coupled with three effective phonon modes with correspondent energies at $62,-139$ and $192 \mathrm{meV}$, which were observed experimentally.
\end{abstract}

\section{Introduction}

Optical and electrical properties of conjugated polymers have been extensively investigated due to their technological interest in optoeletronic devices such as light-emitting devices [1-2]. The progress in the chemical synthesis of poly ( $p$-phenylene vinylene) (PPV), and its derivative, was followed by a similar development in experimental and theoretical electronic structure studies of such materials [3-5]. Optical and electronic properties of conjugated polymer have been investigated by absorption and photoluminescence (PL) spectroscopies [6-7]. In particular, spectroscopic investigations of PPV (and its derivatives) thin films have shown a strong dependence on their structural order, on intrinsic defects, and on molecular aggregations. The conventional procedure to obtain PPV films via the soluble PPVprecursor route should follow a thermal treatment at relatively high temperatures $\left(>250^{\circ} \mathrm{C}\right)$, which can be a source of a great amount of intrinsic defects by thermaloxidation, i. e., the formation of carbonyl groups [8-11]. These defects, which are efficient non-radiative centers and that reduce the effective chain length [12-14], impose a physical limit in the spectra resolution. Typically, the PL spectrum of casting-PPV film presents high electron-phonon coupling with a line-width of zerophonon transition of approximately $250 \mathrm{meV}$ [15]. This value is much higher than the phonon energy measured by infrared and Raman spectroscopies, $100 \mathrm{meV}$ [16-17], indicating that the disordered structure of the solid-film contributes to the line enlargement. As a result, the necessary resolution to measure the phonon energy directly from the emission spectra depends on having films highly ordered and with less intrinsic defects.

In a previous paper we developed an alternative process that allows obtaining PPV converted at relatively low temperature $\left(110{ }^{\circ} \mathrm{C}\right)$, which, in addition, strongly reduced the number of carbonyl groups. Concomitantly, we show that thin films prepared by SA and Langmuir-Blodgett (LB) techniques are much more ordered [15,18-19]. Photoluminescence studies of such films showed an enhancement of the PL spectral resolution. Both, SA and LB films presented well-resolved vibronic PL spectra with a line-width of approximately $65 \mathrm{meV}$, measured at low temperature $(30 \mathrm{~K})$. In the present paper we analyze emission line shapes, measured at $30 \mathrm{~K}$ and at room temperature, of SA-PPV converted at $110{ }^{\circ} \mathrm{C}$, using an empirical model assuming a Gaussian distribution of conjugated segment lengths.

\section{Experimental procedures}

The PPV-precursor, poly(xylyliden tetrahydrothiophenium chloride) (PTHT), was synthesized using the precursor route described in the literature [8]. The concentration of polycation PTHT-solution was $0.16 \mathrm{mg} / \mathrm{ml}$ and the anion of dodecylbenzenesulfonate (DBS) solution was prepared with $10^{-1} \mathrm{M}$ concentration. The solutions were diluted in pure water at $\mathrm{pH}=5.0$. Twenty layers SA-PTHT/DBS were deposited on hydrophilic quartz substrates through alternate immersion in the polycation solution for $1 \mathrm{~min}$ and anion solution for $30 \mathrm{~s}$, and finally dried by $\mathrm{N}_{2}$ flux. The substrate was rinsed in Milli-Q water after each immersion in the solution. The PTHT/DBS films were converted to PPV 
films at low temperature $\left(110{ }^{\circ} \mathrm{C}\right)$ during 30 min under atmospheric conditions.

The optical properties of these films were investigated by UV-Vis and photoluminescence spectroscopies. For absorption experiments we used a Hitachi U-2001 spectrometer. PL measurements in the visible region, were obtained exciting the samples by a 458 $\mathrm{nm}$ line of an $\mathrm{Ar}^{+}$laser with an average excitation of $5 \mathrm{~mW} / \mathrm{cm}^{2}$. The selective excitation spectroscopy (SES) experiments were carried out using a very narrow excitation-line (FWHM $1 \mathrm{~nm}$ ) by dispersing the light of a $450 \mathrm{~W}$ Xe-lamp, which allows the variation of the excitation wavelength (Exc.) from $495 \mathrm{~nm}$ to $527 \mathrm{~nm}$. PL and SES spectra were obtained using a photomultiplier mounted on a $0.5 \mathrm{~m}$ monochromator controlled by the lock-in technique. The temperature could be varied from 30 to $300 \mathrm{~K}$ using a closed-cycle helium cryostat.

\section{Theory analysis}

The line shape of the emission was calculated via Fermi's golden rule containing the coupling between localized molecular states and vibrational modes. The analytic expression was obtained using Condon approximation. The emission coefficient can be calculated considering the electronic transition from excited state $b$ to ground state $a(b \longrightarrow a)$ as in the following expression [20-24]:

$$
I_{n}(\omega)=\frac{2 a_{m} \pi \omega^{3}}{3 c \hbar}\left|\overrightarrow{\mu_{n}}\right|^{2} \int_{-\infty}^{+\infty} d t \exp \left(i t\left(\omega_{n}-\omega\right)-\frac{d^{2} t^{2}}{2}\right) \prod_{j} G_{j}^{*}(t)
$$

where $\overrightarrow{\mu_{n}}$ is the electronic transition moment, $a_{m}$ describes the medium effect, $c$ the speed of light, $\hbar \omega_{n}=$ $E_{b}^{n}-E_{a}^{n}$ is the difference between $\operatorname{LUMO}\left(E_{b}^{n}\right)$ and $\operatorname{HOMO}\left(E_{a}^{n}\right)$ of a conjugate PPV segment with $n$ units, and $d$ represents the inhomogeneous spectral line broad- ening described by a Gaussian distribution. The function $G_{j}^{*}(t)$ is the Frank-Condon factor in harmonic approximation and in the case of pure displacement can expressed as [21,25]:

$$
\prod_{j} G_{j}^{*}(t)=\exp \left[\sum_{j=1}^{N} S_{j}\left\{\left(\bar{n}_{j}+1\right) \exp \left(i t \omega_{j}\right)+\bar{n}_{j}\left(\exp \left(-i t \omega_{j}\right)-1\right)\right\}\right]
$$

where $S_{j}$ is the Huang-Rhys factor, $\omega_{j}$ is the $j^{t h}$-phonon energy, and $\bar{n}_{j}=1 /\left(\exp \left(\hbar \omega_{j} / 2 k T\right)-1\right)$.

\section{Results and discussions}

Figure 1a shows the absorbance spectra of a SA-PPV film as function as the temperature $(10 \mathrm{~K}$ to $300 \mathrm{~K})$. At low temperatures the spectra exhibit well-resolved vibronic structures near the band edge (between 450 and $510 \mathrm{~nm}$ at $10 \mathrm{~K}$ ). As the temperature increases these structures become less resolved, broadening the peaks, owing to the thermal induced structural disorder. Similar behavior is observed in the PL spectra (Fig. 1b). Compared with the absorption of LB-films, the SAspectra present lower absorption in the region of low wavelength. This is an indicative that SA-PPV films converted at low temperature technique [26] exhibits a narrow distribution of the conjugated lengths increasing, therefore, the amount of effective degree of conjugate PPV segments along the polymer chain. Since HOMO-LUMO gaps are lower for large segments the absorption peaks move towards lower energies. On the other hand, the emission peak of SA-PPV at $300 \mathrm{~K}$ is very similar to that of LB-films. However, at low temperature $(30 \mathrm{~K})$ it presents a well-resolved line at 521 $\mathrm{nm}$ with line-width of about $65 \mathrm{meV}$. As the temperature increases the PL spectrum decreases slightly in intensity, owing to thermal activation of non-radiative processes, and is blue-shifted due thermal molecular motions which simulates a reduction of the PPV effective conjugation length. The PL spectrum of LB-PPV film at room temperature is shifted to high energies causing a low Stockes-shift in comparison to SA film (Fig. 1). 

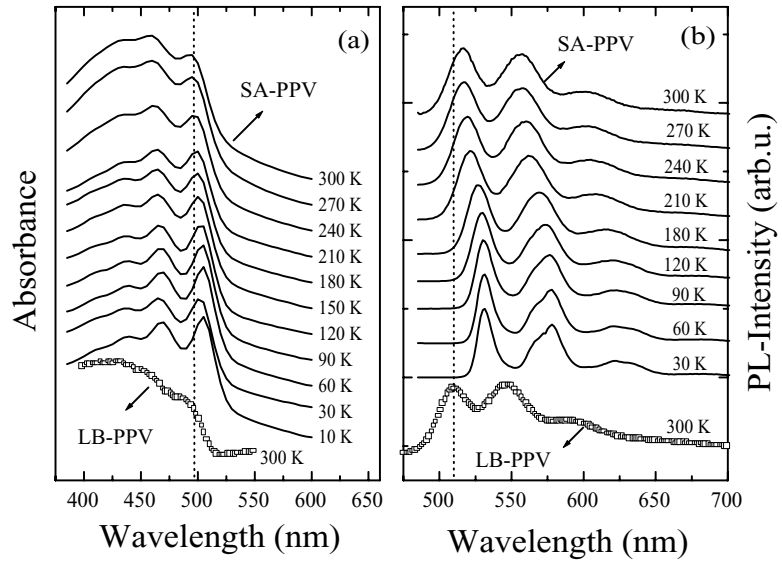

Figure 1. Absorbance (a) and emission (b) spectra of a SAPPV film as function of the temperature. The spectra of a LB-PPV film at room temperature are present for comparison.

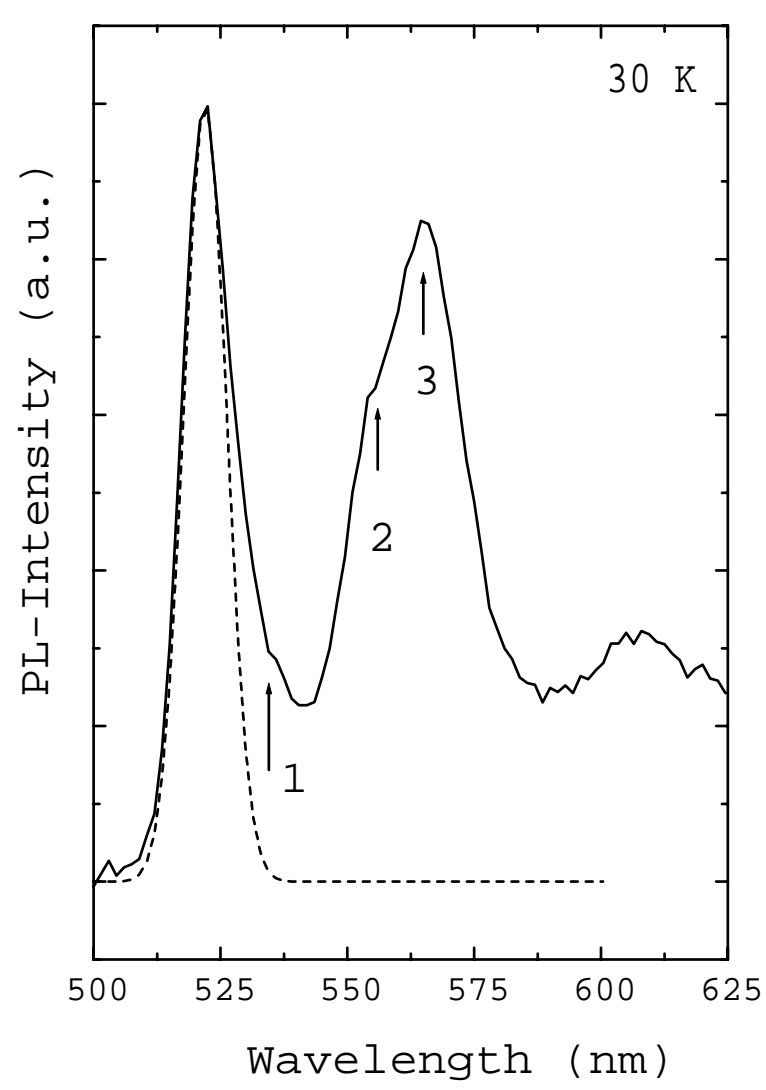

Figure 2. Line shape of PL spectrum at low temperature $(30 \mathrm{~K})$ of SA-PPV film. Three unresolved structures $(1,2$ e 3) are indicated with energy about 535, 555 and $570 \mathrm{~nm}$ with are related with vibrational modes. The zero-phonon line (- - -) is plotted using the equation 1 and $S_{j}=0$.

The PL spectrum at $30 \mathrm{~K}$ of a SA-PPV film is now displayed in Fig. 2 in the $500-625 \mathrm{~nm}$ spectral range, showing in detail the well resolved peak around $521 \mathrm{~nm}$ that is fitted by the zero-phonon transition (dashed line). This fit was obtained assuming a negligible electron-phonon coupling, i.e., the Huang-Rhys parameter $S_{j}$ equal 0 in Eq. 2. The result is a Gaussian function centralized in zero-phonon peak widened by the parameter $d$ (non-homogeneous widening parameter).

Superimposed to the emission spectra it is also observed other small peaks, at 535, 555 and $570 \mathrm{~nm}$ that shall correspond, respectively, to $\omega_{1}=500 \mathrm{~cm}^{-1}(62$ $\mathrm{meV}), \omega_{2}=1170 \mathrm{~cm}^{-1}(139 \mathrm{meV})$ and $\omega_{3}=1550 \mathrm{~cm}^{-1}$ (192 meV). Infrared and Raman measurements [16-17] have identified spectral features of PPV vibronic modes of C-H plus C-C ring out-of-plane bend (522 and 555 $\mathrm{cm}^{-1}$ ), C-H ring in-plane-bend (1005, 1135 and 1174 $\left.\mathrm{cm}^{-1}\right)$ and $\mathrm{C}-\mathrm{C}$ ring stretch $(1518,1543,1550$ and 1584 $\mathrm{cm}^{-1}$ ). Then, we used in our calculations these three effective phonons coupled with the electronic transitions as parameters, for which $S_{1}, S_{2}$ and $S_{3}$ are the HuangRhys factors respectively.

Figure 3 shows PL spectra obtained from SES measurements, carried out at $30 \mathrm{~K}$, of a SA-PPV sample. The sharp peaks in the left are the excitation lines, which was varied from $495 \mathrm{~nm}$ to $527 \mathrm{~nm}$. The excitation energy was scanned over the zero-phonon spectral region selecting only long conjugated PPV segments. As the excitation energy goes through the zero-phonon transition $(521 \mathrm{~nm})$, two spectral lines, at $555 \mathrm{~nm}$ and $570 \mathrm{~nm}$, become well defined showing the existence of two effective modes that were not resolved in the normal PL spectra of Fig. 2.

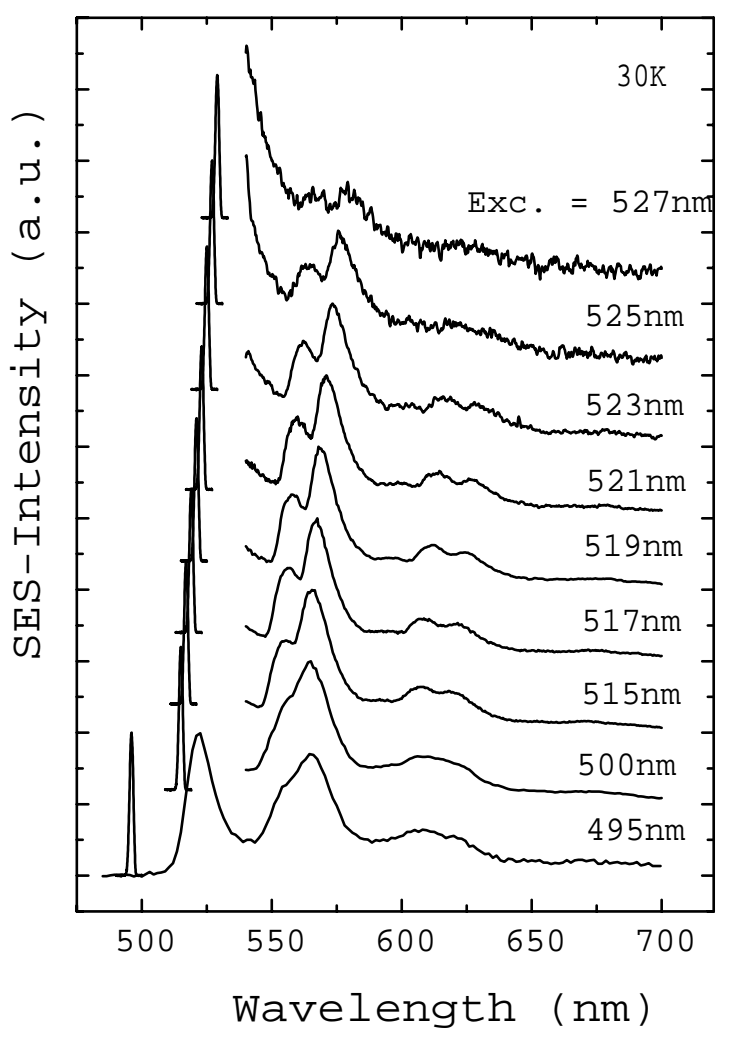

Figure 3. SES experiment with excitation (Exc.) varing from $495 \mathrm{~nm}$ to $527 \mathrm{~nm}$ at low temperature $(30 \mathrm{~K})$ of a SAPPV. A definition in the first phonon replica is observed. 
The theoretical model, expressed by Eq. 1, was applied to fit the PL spectra presented in Fig. 1. In the fitting, we fixed, based on the experimental result of Fig. 2, the phonon frequencies $\left(500 \mathrm{~cm}^{-1}, 1170\right.$ $\mathrm{cm}^{-1}$ and $1550 \mathrm{~cm}^{-1}$ ), but we varied the Huang-Rhys parameters $\left(S_{j}\right)$, the homogeneous widening $(d)$ and the zero-phonon position $\left(\omega_{n}\right)$. Figure 4 shows that the theoretical model presents a reasonable agreement with the experimental data (at $30 \mathrm{~K}$ ). The contribution of each vibrational mode is also shown in the Fig. 4. The used parameters were: the width $d$ is equal (580 $\left.\mathrm{cm}^{-1}\right), \omega_{n}$ equal $2.34 \mathrm{eV}$, and the Huang-Rhys parameters $S_{1}=0.14, S_{2}=0.48$, and $S_{3}=0.80$. The two first parameters, $d$ and $\omega_{n}$ were extracted from the experimental results. The relatively low Ruang-Hys parameter values $\left(S_{j}<1\right)$ confirm that SA-PPV has rather long conjugated segments and low molecular disorder [24]. Considering the efficient carrier migration mechanism to lower energy states (HOMO and LUMO molecular states), the radiative recombination occurs at high PPV conjugate segments ( 10 units) [27].

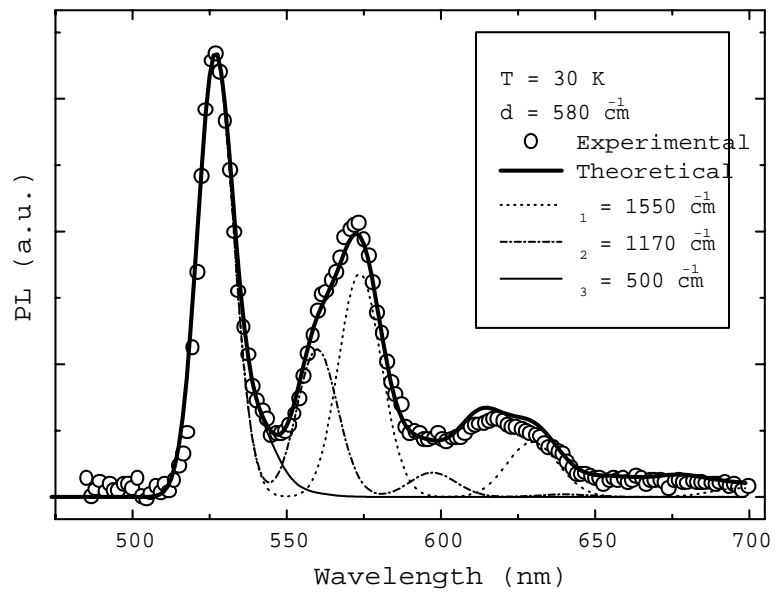

Figure 4. PL spectrum at low temperature $(30 \mathrm{~K})$ of SAPPV film $(-\mathrm{O}-)$ and theoretical spectrum $(-)$ considering the contribution of three effective phonons at $500 \mathrm{~cm}^{-1}$ $(-), 1170 \mathrm{~cm}^{-1}(---)$ and $1550 \mathrm{~cm}^{-1}(\cdots)$.

Similar fitting were carried out with the spectrum obtained at $300 \mathrm{~K}$, shown in Fig. 5. In this fitting the adjusted parameters were: $S_{1}=0.22, S_{2}=0.60$ and $S_{3}=0.85$, whereas $d$ equal to $840 \mathrm{~cm}^{-1}$ and $\omega_{n}$ is equal $2.39 \mathrm{eV}$. The broadening of the zero-phonon linewidth $d$ and the $\omega_{n}$-blue-shift of $50 \mathrm{meV}$ reflects the thermal disorder induced by the temperature. The enhancement of $S_{1}$ and $S_{2}$ values shows that the electron-phonon coupling also depends on the temperature, which indicates that the electronic molecular states are more sensitive to the ring vibration (phenylene ring) in the PPV unit. Since $\omega_{1}$ and $\omega_{2}$ are associated, respectively, with the bend vibration of the ring out-of-plane and the ring inplane-bend [16], we may expect that these phenomenon undergoes intermolecular interactions, being higher for high temperatures.

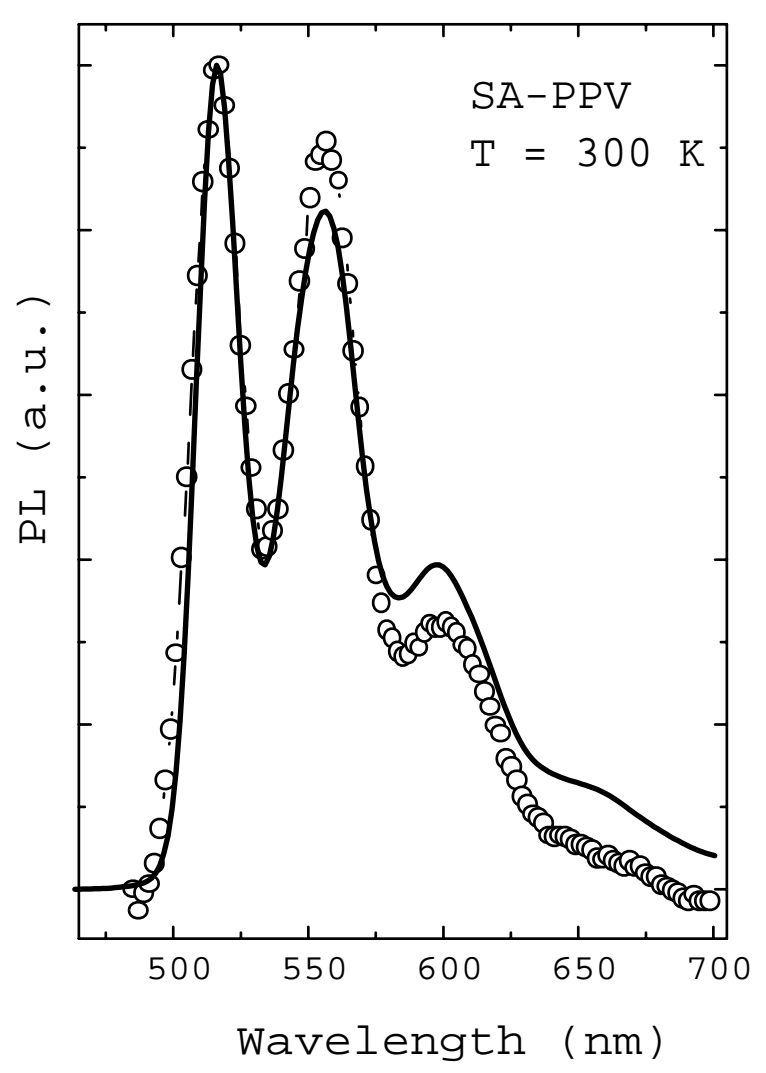

Figure 5. PL spectrum at room temperature $(300 \mathrm{~K})$ of SA-PPV film (-O-) and theoretical spectrum (-

\section{Conclusions}

In this paper we show that SA-PPV films display absorption and emission curves with high resolution, mainly at low temperatures, allowing a quantitative study of the emission spectrum considering its vibronic structures. In addition, by the use of the SES technique we were able to resolve the vibrational mode associated with the first replica of zero phonon transition. Calculations of electronic transition rates were obtained by a model based on the Condon approximation taking into account electron-phonon coupling and a distribution in lengths of conjugated lengths along the polymeric chain. By the analysis of the HuangRhys parameters and the zero-phonon line-width and its position in the spectrum we fitted, with great accuracy, the experimental emission curve at low temperature. The small Huang-Rhys coupling-parameters (smaller than the unity) confirms the quality of the produced SA-PPV films when compared to that made by conventional casting procedure. The slight difference observed between the theoretical and the experimental curves in Fig. 5 indicates that other interactions need to be considered, as, for example, interchain interactions and the effect of clusters of molecules [14,28-30]. 


\section{Acknowledgement}

The financial support from Brazilian agency Fundação de Apoio à Pesquisa do Estado de São Paulo (FAPESP) is acknowledged.

\section{References}

[1] R. H. Friend, R. W. Gymer, A. B. Holmes, J. H. Burroughes, R. N. Marks, C. Taliani, D. D. C. Bradley, D. A. dos Santos, J. L. Bredas, M. Logdlund, and W. R. Salaneck, Nature 397, 121 (1999).

[2] M. T. Bernius, M. Inbasekaran, J. O'Brien, and W. Wu, Adv. Mat 12, 1737 (2000).

[3] S. C. Graham, D. D. Bradley, R. H. Friend, and C. W. Spangler, Synth. Met. 41-43, 1277 (1991).

[4] T. W. Hagler, K. Pakbaz, and A. J. Heeger, Phys. Rev. B 44, 8652 (1991).

[5] K. Pichler, D. A. Halliday, D. D. Bradley, P. L. Burn, R. H. Friend, and A. B. Holmes, J. Phys.: Condens. Matter 5, 7155 (1993).

[6] K. Pakbaz, C. H. Lee, A. J. Heeger, T. W. Hagler, and D . Mcbranch, Synth. Met. 64, 295 (1994).

[7] J. Obrzut and F. E. Karasz, J. Chem. Phys. 87, 2349 (1987).

[8] D. A. Halliday, P. L. Burn, R. H. Friend, and A. B. Holmes, J. Chem. Soc. Chem. Commun. 22, 1685 (1992).

[9] B. H. Cumpston and K. F. Jensen, TRIP 4, 151 (1996).

[10] F. Papadimitrakopoulos, M. Yan, L. J. Rothberg, H. E. Katz, E. A. Chandross, and M. E. Galvin, Mol. Crys. Liq. Crys. 256, 663 (1994).

[11] W. Holzer, A. Penzkofer, M. Pichlmaier, D. D. C. Bradley, and W. J. Blau, Chem. Phys. 248, 273 (1999).

[12] L. J. Rothberg, M. Yan, F. Papadimitrakopoulos, M. E. Galvin, E. W. Kwock, and T. M. Miller, Synth. Met. 80, 41 (1996).

[13] R. Kersting, U. Lemmer, R. F. Mahart, K. Leo, H. Kurz, H. Bässler, and E. O. Göbel, Phys. Rev. Lett. 70, 3820 (1993).

[14] M. Yan, L. J. Rothberg, F. Papadimitrakopoulos, M. E. Galvin, and T. M. Miller, Phys. Rev. Lett. 73, 744 (1994).
[15] A. Marletta, D. Gonçalves, O.N. Oliveira Jr., R.M. Faria, and F.E.G. Guimarães, Macromolecules 28, 7107 (1995).

[16] D. Rakvié, R. Kostié, L. A. Gribov, and I. E. Davidova, Phys. Rev. B 41, 10744 (1990).

[17] S. Lefrant, E. Perrin, and J. P. Buisson, Synth. Met. 29, E91 (1989).

[18] A. Marletta, D. Gonçalves, O. N. Oliveira Jr., R. M. Faria, and F. E. G. Guimarães, Synth. Met. 119, 1447 (2001).

[19] A. Marletta, D. Gonçalves, O. N. Oliveira Jr., R. M. Faria, and F. E. G. Guimarães, Synth. Met. 119, 629 (2001).

[20] S. H. Lin, J. Chem. Phys. 44, 3759 (1966).

[21] A. D. Brailford and T. Y. Chang, J. Chem. Phys. 53, 3109 (1970).

[22] J. Yu, W. S. Fann, F. J. Kao, D. Y. Yang, and S. H. Lin, Synth. Met. 66, 143 (1994).

[23] J. Yu, M. Hayashi, S. H. Lin, K. K. Liang, J. H. Hsu, W. S. Fann, C. I. Chao, K. R. Chuang, and S. A. Chen, Synth. Met. 82, 159 (1996).

[24] R. Chang, J. H. Hsu, W. S. Fann, K. K. Liang, C. H. Chang, M. Hayashi, J. Yu, S. H. Lin E. C. Chang, K. R. Chuang, and S. A. Chen, Chem. Phys. Lett. 317, $142(2000)$.

[25] R. Chang, J. C. Chang, R. Chang, J. H. Hsu, W. S. Fann, M. Hayashi, J. Yu, J. C. Jiang, S. H. Lin, Y. Z. Lee, K. R. Chuang, and S. A. Chen, Synth. Met. 106, 139 (1999).

[26] A. Marletta, D. Gonçalves, O.N. Oliveira Jr., R.M. Faria, and F.E.G. Guimarães, Adv. Mat. 12, 69 (2000).

[27] D. G. Cahill, M. Katiyar, and J. R. Abeldeson, Phys. Rev. B 50, 6077 (1994)

[28] Y. Shi, J. Liu, and Y. Yang, J. Appl. Phys. 87, 4254 (2000).

[29] J. W. Blatchford, S. W. Jessen, L. B. Lin, T. L. Gustafson, D. K. Fu, H. L. Wang, T. M. Swager, A. G. MacDiarmid, and A. J. Epstein, Phys. Rev. B 54, 9180 (1996).

[30] T. Q. Nguyen, V. Doan, and B. J. Schwartz, J. Chem. Phys. 110, 4068 (1999). 